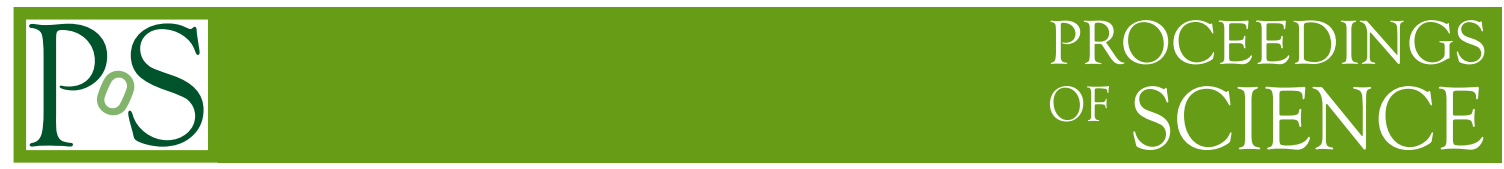

\title{
Cosmological brane solutions
}

\author{
Kunihito Uzawa \\ Department of Physics, Kindai University, Higashi-Osaka, Osaka 577-8502, Japan \\ E-mail: uzawa@phys.kindai.ac.jp
}

\begin{abstract}
The recent discovery of an explicit dynamical description of $p$-branes makes it possible to investigate the existence of intersection of such objects. We generalize those solutions depending on the overall transverse space coordinates and time to those which depend also on the relative transverse space and satisfy new intersection rules. We give classification of these dynamical intersecting brane solutions involving two branes, and discuss the application of these solutions to cosmology and show that these give Friedmann-Lemaitre-Robertson-Walker cosmological solutions.
\end{abstract}

25th Texas Symposium on Relativistic Astrophysics

December 6-10, 2010

Heidelberg, Germany 


\section{Contents}

$\begin{array}{lr}\text { 1. Introduction } & 2\end{array}$

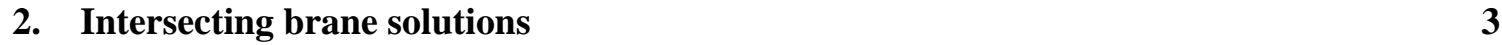

$\begin{array}{lr}\text { 3. Discussions } & 7\end{array}$

\section{Introduction}

The dynamics of the brane world model in five or six dimensions have been much explored because of the possible cosmological and phenomenological interests. Although some results have recently emerged on the applications of the solutions in higher-dimensional supergravity to the brane world cosmology, e.g., in [1], the construction of the cosmological model in string theory is much less extensive. One motivation for the present work is to improve this situation. For this purpose, it is first necessary to construct dynamical brane solutions depending on the time as well as space coordinates.

It has already been known [2,3] that dynamical brane solutions arise when the gravity is coupled not only to a single gauge field but also to several combinations of scalars and forms, as generalization of the static intersecting brane solutions in the supergravity. Here we construct dynamical brane solutions by generalizing these static solutions to a dynamical one. The first class of dynamical solutions we study in this paper has the dependence on the time as well as overall transverse space coordinates in the metric and obeys the well-known intersection rules. However, it has also been known for some time that some static intersecting brane solutions may not follow these intersection rules. These intersecting brane solutions are derived for the case when the branes depend on the relative transverse directions of the intersecting branes.

Our goal is to exhaust and classify all two-intersecting-brane solutions which depend on the time and (relative) transverse dimensions and to study their applications to the cosmological evolutions, in particular in the ten-dimensional string theory and eleven-dimensional supergravity theory. We first find cosmological solutions for possible intersections including the above exceptional cases for two intersecting branes by extending the similar solutions obeying the usual intersection rules [2,3]. Our results on the dynamical branes are given for general cases of arbitrary dimensions and forms, but in their applications to cosmology, we mainly focus on the dynamical branes in tenand eleven-dimensional supergravities because these are the most important low-energy effective theories of superstrings. 


\section{Intersecting brane solutions}

We consider a $D$-dimensional theory composed of the metric $g_{M N}$, dilaton $\phi$, and the antisymmetric tensor fields of rank $\left(p_{r}+2\right)$ and $\left(p_{s}+2\right)$ :

$$
S=\frac{1}{2 \kappa^{2}} \int\left[R * \mathbf{1}-\frac{1}{2} d \phi \wedge * d \phi-\frac{1}{2} \frac{\mathrm{e}^{\varepsilon_{r} c_{r} \phi}}{\left(p_{r}+2\right) !} F_{\left(p_{r}+2\right)} \wedge * F_{\left(p_{r}+2\right)}-\frac{1}{2} \frac{\mathrm{e}^{\varepsilon_{s} c_{s} \phi}}{\left(p_{s}+2\right) !} F_{\left(p_{s}+2\right)} \wedge * F_{\left(p_{s}+2\right)}\right],
$$

where $\kappa^{2}$ is the $D$-dimensional gravitational constant, $*$ is the Hodge operator in the $D$-dimensional spacetime, $F_{(n)}$ is an $n$-form field strength, and $c_{I}, \varepsilon_{I}(I=r, s)$ are constants given by

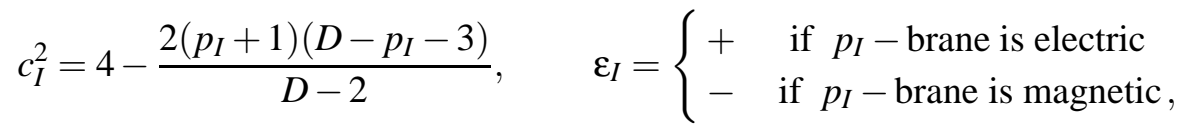

To solve these field equations, we assume that the $D$-dimensional metric takes the form

$$
d s^{2}=h_{r}^{\alpha} h_{s}^{\beta}\left[h_{r}^{-1} h_{s}^{-1} q_{\mu v}(\mathrm{X}) d x^{u} d x^{v}+h_{s}^{-1} \gamma_{i j}\left(\mathrm{Y}_{1}\right) d y^{i} d y^{j}+h_{r}^{-1} w_{m n}\left(\mathrm{Y}_{2}\right) d v^{m} d v^{n}+u_{a b}(\mathrm{Z}) d z^{a} d z^{b}\right],
$$

where $q_{\mu v}, \gamma_{i j}, w_{m n}$, and $u_{a b}$ are the metrics depending only on $x^{\mu}, y^{i}, v^{m}$, and $z^{a}$ coordinates of dimensions $(p+1),\left(p_{s}-p\right),\left(p_{r}-p\right)$, and $\left(D+p-p_{r}-p_{s}-1\right)$, respectively. The parameters $\alpha$ and $\beta$ in the metric (2.3) are given as $\alpha=\left(p_{r}+1\right)(D-2)^{-1}, \beta=\left(p_{s}+1\right)(D-2)^{-1}$. Here we suppose that $p_{s}\left(p_{r}\right)$-brane extends along $\mathrm{X}$ and $\mathrm{Y}_{1}\left(\mathrm{Y}_{2}\right)$ spaces.

The $D$-dimensional metric (2.3) implies that the $p$-brane solutions are characterized by a function which depends on the coordinates transverse to the brane as well as the worldvolume coordinate. For the configurations of two branes, we should sort the coordinates in three sets and the powers of harmonic functions are different for each set of coordinates according to the intersection rules. One set of the coordinates is the overall worldvolume coordinates, which are common to the two branes. The others are overall transverse coordinates and the last are the relative transverse coordinates, which are transverse to only one of the two branes.

The field equations of intersecting branes allow for the following three kinds of possibilities on $p_{r^{-}}$and $p_{s}$-branes in $D$ dimensions [4]:

(I)Both $h_{r}$ and $h_{s}$ depend on the overall transverse coordinates: $h_{r}=h_{r}(x, z), h_{s}=h_{s}(x, z)$.

(II)Only $h_{s}$ depends on the overall transverse coordinates, but the other $h_{r}$ does on the corresponding relative coordinates: $h_{r}=h_{r}(x, y), h_{s}=h_{s}(x, z)$.

(III)Each of $h_{r}$ and $h_{s}$ depends on the corresponding relative coordinates: $h_{r}=h_{r}(x, y), h_{s}=$ $h_{s}(x, v)$.

In the following, we consider intersections where each participating brane corresponds to an independent harmonic function in the solution and derive the dynamical intersecting brane solution in $D$ dimensions satisfying the above conditions.

For completeness, let us first consider case (I) though this has been already discussed in [3]. For this class, we assume that the scalar field $\phi$ and the gauge field strengths are given as

$$
\begin{aligned}
\mathrm{e}^{\phi} & =h_{r}^{\varepsilon_{r} c_{r} / 2} h_{s}^{\varepsilon_{s} c_{s} / 2}, \\
F_{\left(p_{r}+2\right)} & =d\left[h_{r}^{-1}(x, z)\right] \wedge \Omega(\mathrm{X}) \wedge \Omega\left(\mathrm{Y}_{2}\right), \quad F_{\left(p_{s}+2\right)}=d\left[h_{s}^{-1}(x, z)\right] \wedge \Omega(\mathrm{X}) \wedge \Omega\left(\mathrm{Y}_{1}\right),
\end{aligned}
$$


where $\Omega(\mathrm{X}), \Omega\left(\mathrm{Y}_{1}\right)$, and $\Omega\left(\mathrm{Y}_{2}\right)$ denote the volume forms of dimensions $(p+1),\left(p_{s}-p\right)$, and $\left(p_{r}-p\right)$, respectively. Under the assumptions (2.3), (2.4), and (2.5), the field equations lead to [3]

$$
\begin{aligned}
& R_{\mu v}(\mathrm{X})=0, \quad R_{i j}\left(\mathrm{Y}_{1}\right)=0, \quad R_{m n}\left(\mathrm{Y}_{2}\right)=0, \quad R_{a b}(\mathrm{Z})=0, \\
& h_{r}=h_{0}(x)+h_{1}(z), \quad h_{s}=k_{0}(x)+k_{1}(z), \\
& D_{\mu} D_{v} h_{0}=0, \quad \triangle_{\mathrm{Z}} h_{1}=0, \quad \triangle_{\mathrm{Z}} h_{s}=0, \quad \text { for } \partial_{\mu} h_{s}=0, \\
& D_{\mu} D_{v} k_{0}=0, \quad \triangle_{\mathrm{Z}} k_{1}=0, \quad \triangle_{\mathrm{Z}} h_{r}=0, \quad \text { for } \partial_{\mu} h_{r}=0,
\end{aligned}
$$

where $D_{\mu}$ is the covariant derivative with respect to the metric $q_{\mu v}$, and $\triangle_{\mathrm{Z}}$ is the Laplace operators on the space of $\mathrm{Z}$, and $R_{\mu}(\mathrm{X}), R_{i j}\left(\mathrm{Y}_{1}\right), R_{m n}\left(\mathrm{Y}_{2}\right)$, and $R_{a b}(\mathrm{Z})$ are the Ricci tensors of the metrics, $q_{\mu v}(\mathrm{X}), \gamma_{i j}\left(\mathrm{Y}_{1}\right), w_{m n}\left(\mathrm{Y}_{2}\right)$, and $u_{a b}(\mathrm{Z})$, respectively, and we used the intersection rule $\chi=0$ [3]

$$
\chi=p+1-\frac{\left(p_{r}+1\right)\left(p_{s}+1\right)}{D-2}+\frac{1}{2} \varepsilon_{r} \varepsilon_{s} c_{r} c_{s} .
$$

If $F_{\left(p_{r}+2\right)}=0$ and $F_{\left(p_{s}+2\right)}=0$, the functions $h_{1}$ and $k_{1}$ become trivial.

As a special example, let us consider the case $q_{\mu v}=\eta_{\mu v}, \gamma_{i j}=\delta_{i j}, w_{m n}=\delta_{m n}, u_{a b}=\delta_{a b}$, where $\eta_{\mu v}$ is the $(p+1)$-dimensional Minkowski metric and $\delta_{i j}, \delta_{m n}, \delta_{a b}$ are the $\left(p_{s}-p\right)-,\left(p_{r}-p\right)$-, and $\left(D+p-p_{r}-p_{s}-1\right)$-dimensional Euclidean metrics, respectively. For $\partial_{\mu} h_{s}=0$, the solution for $h_{r}$ and $h_{s}$ can be obtained explicitly as

$$
h_{r}(x, z)=A_{\mu} x^{\mu}+B+\sum_{l} \frac{M_{l}}{\left|z-z_{l}\right|^{D+p-p_{r}-p_{s}-3}}, \quad h_{s}(z)=C+\sum_{c} \frac{M_{c}}{\left|z-z_{c}\right|^{D+p-p_{r}-p_{s}-3}},
$$

where $A_{\mu}, B, C, M_{l}$, and $M_{c}$ are constant parameters, and $z_{l}$ and $z_{c}$ are constant vectors representing the positions of the branes. We can also choose the solution in which the $p_{s}$-brane part depends on both $x$ and $z$.

Now we discuss the intersecting brane solutions in eleven-dimensional supergravity and in ten-dimensional string theories. For the M-branes in eleven-dimensional supergravity, there is 4form field strength without dilaton, so the intersection rule $\chi=0$ gives $p=\left(p_{r}+1\right)\left(p_{s}+1\right) / 9-1$, where $p$ denotes the number of overlapping dimensions of the $p_{r}$ and $p_{s}$ branes. Then we get the intersections involving the M2 and M5-branes [3]

$$
\mathrm{M} 2 \cap \mathrm{M} 2=0, \quad \mathrm{M} 2 \cap \mathrm{M} 5=1, \quad \mathrm{M} 5 \cap \mathrm{M} 5=3 .
$$

For the ten-dimensional string theories, the couplings to dilaton for the RR-charged D-branes are given by $\varepsilon_{r} c_{r}=\left(3-p_{r}\right) / 2, \varepsilon_{s} c_{s}=\left(3-p_{s}\right) / 2$ The condition $\chi=0$ then gives $p=\left(p_{r}+p_{s}-\right.$ 4) $/ 2$. The intersections for the D-branes are thus given by

$$
\mathrm{D} p_{r} \cap \mathrm{D} p_{s}=\left(p_{r}+p_{s}\right) / 2-2 \text {. }
$$

We finally consider the intersections for NS-branes. The parameters $c_{r}$ for fundamental string (F1) and solitonic 5-brane are $\varepsilon_{1} c_{1}=-1$ (for F1) and $\varepsilon_{5} c_{5}=1$ (for NS5), respectively. Then the intersections involving the F1 and NS5-branes are [3]

$$
\mathrm{F} 1 \cap \mathrm{NS} 5=1, \quad \mathrm{NS} 5 \cap \mathrm{NS} 5=3, \quad \mathrm{~F} 1 \cap \mathrm{D} \bar{p}=0, \quad \mathrm{D} \bar{p} \cap \mathrm{NS} 5=\bar{p}-1, \quad 1 \leq \bar{p} \leq 6 .
$$


There is no solution for the F1-F1 and D0-NS5 intersecting brane systems because the numbers of space dimensions for each pairwise overlap are negative by the intersection rule.

We next consider case (II). We take the following ansatz for the scalar field $\phi$ and the gauge field strengths:

$$
\begin{aligned}
\mathrm{e}^{\phi} & =h_{r}^{\varepsilon_{r} c_{r} / 2} h_{s}^{\varepsilon_{s} c_{s} / 2}, \\
F_{\left(p_{r}+2\right)} & =d\left[h_{r}^{-1}(x, y)\right] \wedge \Omega(\mathrm{X}) \wedge \Omega\left(\mathrm{Y}_{2}\right), \quad F_{\left(p_{s}+2\right)}=d\left[h_{s}^{-1}(x, z)\right] \wedge \Omega(\mathrm{X}) \wedge \Omega\left(\mathrm{Y}_{1}\right),
\end{aligned}
$$

where $\Omega(\mathrm{X}), \Omega\left(\mathrm{Y}_{1}\right)$, and $\Omega\left(\mathrm{Y}_{2}\right)$ are the volume forms of dimensions $(p+1),\left(p_{s}-p\right)$, and $\left(p_{r}-p\right)$, respectively. Since we use the same procedure as in case (I), we can derive the intersection rule $\chi=0$ from the field equations. For $\chi=0$, it is easy to show that the field equations reduce to

$$
\begin{aligned}
& R_{\mu v}(\mathrm{X})=0, \quad R_{i j}\left(\mathrm{Y}_{1}\right)=0, \quad R_{m n}\left(\mathrm{Y}_{2}\right)=0, \quad R_{a b}(\mathrm{Z})=0, \\
& h_{r}=h_{0}(x)+h_{1}(y), \quad h_{s}=h_{s}(z), \quad D_{\mu} D_{v} h_{0}=0, \quad \triangle_{\mathrm{Y}_{1}} h_{1}=0, \quad \triangle_{\mathrm{Z}} h_{s}=0, \quad \partial_{\mu} h_{s}=0,
\end{aligned}
$$

where $\triangle_{\mathrm{Y}_{1}}$ is the Laplace operators on the space of $\mathrm{Y}_{1}$. If $F_{\left(p_{r}+2\right)} \neq 0$ and $F_{\left(p_{s}+2\right)} \neq 0$, the functions $h_{1}$ and $k_{1}$ are nontrivial.

Let us consider the following case in more detail: $q_{\mu v}=\eta_{\mu v}, \gamma_{i j}=\delta_{i j}, w_{m n}=\delta_{m n}, u_{a b}=\delta_{a b}$, where $\eta_{\mu v}$ is the $(p+1)$-dimensional Minkowski metric and $\delta_{i j}, \delta_{m n}, \delta_{a b}$ are the $\left(p_{s}-p\right)-,\left(p_{r}-p\right)$ , and $\left(D+p-p_{r}-p_{s}-1\right)$-dimensional Euclidean metrics, respectively. For $\partial_{\mu} h_{s}=0$, the solution for $h_{r}$ and $h_{s}$ can be obtained explicitly as

$$
h_{r}(x, y)=A_{\mu} x^{\mu}+B+\sum_{l} \frac{M_{l}}{\left|\boldsymbol{y}-\boldsymbol{y}_{l}\right|^{p_{s}-p-2}}, \quad h_{s}(z)=C+\sum_{c} \frac{M_{c}}{\left|\boldsymbol{z}-\boldsymbol{z}_{c}\right|^{D+p-p_{r}-p_{s}-3}},
$$

where $A_{\mu}, B, C, \boldsymbol{y}_{l}, z_{c}, M_{l}$, and $M_{c}$ are constant parameters.

One can easily get the solution for $\partial_{\mu} h_{r}=0$ and $\partial_{\mu} h_{s} \neq 0$ if the roles of $\mathrm{Y}_{1}$ and $\mathrm{Y}_{2}$ are exchanged. Since the dynamical solution (2.19) obeys the same intersection rule $\chi=0$, the intersections of M-branes in eleven-dimensional supergravity and D-branes in ten-dimensional string theories are given as (2.12), (2.13), and (2.14).

Finally, we consider case (III). We assume that the scalar field $\phi$ and the gauge field strengths are given as

$$
\begin{aligned}
\mathrm{e}^{\phi} & =h_{r}^{\varepsilon_{r} c_{r} / 2} h_{s}^{\varepsilon_{s} c_{s} / 2}, \\
F_{\left(p_{r}+2\right)} & =h_{s} d\left[h_{r}^{-1}(x, y)\right] \wedge \Omega(\mathrm{X}) \wedge \Omega\left(\mathrm{Y}_{2}\right), \quad F_{\left(p_{s}+2\right)}=h_{r} d\left[h_{s}^{-1}(x, v)\right] \wedge \Omega(\mathrm{X}) \wedge \Omega\left(\mathrm{Y}_{1}\right),
\end{aligned}
$$

where $\Omega(\mathrm{X}), \Omega\left(\mathrm{Y}_{1}\right)$, and $\Omega\left(\mathrm{Y}_{2}\right)$ denote the volume $(p+1)-,\left(p_{s}-p\right)$-, and $\left(p_{r}-p\right)$-forms, respectively.

Under the assumption, the field equations give the intersection rule $\chi=-2$. This is different from the usual rule applicable to the cases (I) and (II). Upon using the intersection rule $\chi=-2$, it is easy to show that the field equations reduce to

$$
\begin{aligned}
& R_{\mu v}(\mathrm{X})=0, \quad R_{i j}\left(\mathrm{Y}_{1}\right)=0, \quad R_{m n}\left(\mathrm{Y}_{2}\right)=0, \quad R_{a b}(\mathrm{Z})=0, \\
& h_{r}=h_{0}(x)+h_{1}(y), \quad h_{s}=k_{0}(x)+k_{1}(v), \\
& D_{\mu} D_{v} h_{0}=0, \quad \triangle_{\mathrm{Y}_{1}} h_{1}=0, \quad \triangle_{\mathrm{Y}_{2}} h_{s}=0, \quad \text { for } \partial_{\mu} h_{s}=0 \\
& D_{\mu} D_{v} k_{0}=0, \quad \triangle_{\mathrm{Y}_{1}} h_{r}=0, \quad \triangle_{\mathrm{Y}_{2}} k_{1}=0, \quad \text { for } \partial_{\mu} h_{r}=0 .
\end{aligned}
$$


where $\triangle_{\mathrm{Y}_{1}}$ and $\triangle_{\mathrm{Y}_{2}}$ are the Laplace operators on the spaces of $\mathrm{Y}_{1}$ and $\mathrm{Y}_{2}$, respectively. The functions $h_{1}$ and $k_{1}$ are nontrivial for $F_{\left(p_{r}+2\right)} \neq 0$ and $F_{\left(p_{s}+2\right)} \neq 0$.

Now we consider the case $q_{\mu \nu}=\eta_{\mu v}, \gamma_{i j}=\delta_{i j}, w_{m n}=\delta_{m n}, u_{a b}=\delta_{a b}$, where $\eta_{\mu v}$ is the $(p+1)$ dimensional Minkowski metric and $\delta_{i j}, \delta_{m n}, \delta_{a b}$ are the $\left(p_{s}-p\right)-,\left(p_{r}-p\right)$-, and $\left(D+p-p_{r}-\right.$ $\left.p_{s}-1\right)$-dimensional Euclidean metrics, respectively. For $\partial_{\mu} h_{s}=0$, the solution for $h_{r}$ and $h_{s}$ can be obtained explicitly as

$$
h_{r}(x, y)=A_{\mu} x^{u}+B+\sum_{l} \frac{M_{l}}{\left|\boldsymbol{y}-\boldsymbol{y}_{l}\right|^{p_{s}-p-2}}, \quad h_{s}(v)=C+\sum_{c} \frac{M_{c}}{\left|\boldsymbol{v}-\boldsymbol{v}_{c}\right|^{p_{r}-p-2}},
$$

where $A_{\mu}, B, C, \boldsymbol{y}_{l}, \boldsymbol{v}_{c}, M_{l}$, and $M_{c}$ are constant parameters. We can also get the solution in which the function $h_{s}$ depends on both $x$ and $v$.

Let us consider the intersecting brane solutions in eleven-dimensional supergravity and in tendimensional string theories. We first discuss the intersections of M-branes in eleven-dimensional supergravity. The intersection rule $\chi=-2$ leads to

$$
p=\frac{\left(p_{r}+1\right)\left(p_{s}+1\right)}{9}-3
$$

Then we get the intersection involving the M5-brane M5 $\cap$ M5 = 1. Equation Eq. (2.27) tells us that the numbers of intersection for M2-M2 and M2-M5 branes are negative, which means that there is no intersecting solution of these brane systems.

Next we consider the intersection in the ten-dimensional string theory. The couplings to dilaton for the RR-charged D-branes are $\varepsilon_{r} c_{r}=\left(3-p_{r}\right) / 2, \varepsilon_{s} c_{s}=\left(3-p_{s}\right) / 2$, and the condition $\chi=$ -2 is expressed as $p=\left(p_{r}+p_{s}-8\right) / 2$. The intersections for the RR-charged D-branes are thus given by $\mathrm{D} p_{r} \cap \mathrm{D} p_{s}=\left(p_{r}+p_{s}\right) / 2-4$. We finally consider the intersections for NS-branes. The parameters $c_{r}$ for fundamental string (F1) and solitonic 5-brane are $\varepsilon_{1} c_{1}=-1$ for $\mathrm{F} 1$ and $\varepsilon_{5} c_{5}=1$ for NS5, respectively. Then the intersection with F1-brane is forbidden by the intersection rule. The intersections involving the NS5-branes are NS5 $\cap$ NS5 $=1$, and $\mathrm{D} \bar{p} \cap \mathrm{NS} 5=\bar{p}-3, \quad(3 \leq \bar{p} \leq 8)$. There is no brane solution involving other intersections because the numbers of space dimensions for each pairwise overlap become negative by the intersection rule.

We list the FLRW cosmological solutions with an isotropic and homogeneous three-space for the solutions in [4] for M-branes, D-branes, F1 and NS5-branes. Since the time dependence in the metric comes from only one brane in the intersections, the obtained expansion law is simple. In order to find an expanding universe, one may have to compactify the vacuum bulk space as well as the brane worldvolume. If we introduce the cosmic time $\tau$, we find that the fastest expanding case in the Jordan frame has the power $a \propto \tau^{7 / 15}$, which is too small to give a realistic expansion law like that in the matter dominated era $\left(a \propto \tau^{2 / 3}\right)$ or that in the radiation dominated era $\left(a \propto \tau^{1 / 2}\right)$, where $a$ is the scale factor of our Universe. When we compactify the extra dimensions and go to the four-dimensional Einstein frame, the power exponents are different depending on how we compactify the extra dimensions even within one solution. We give the power exponent of the fastest expansion of our four-dimensional universe in the Einstein frame in [4]. We again see that the expansion is too small. Hence, we have to conclude that in order to find a realistic expansion of the universe in this type of models, one has to include additional "matter" fields on the brane. 


\section{Discussions}

We have derived intersecting dynamical brane solutions and discussed their dynamics in the ten- and eleven-dimensional supergravity models. These solutions are obtained by replacing a constant $A$ in the warp factor $h=A+h_{1}(y)$ of a supersymmetric solution by a function $h_{0}(x)$ of the coordinates $x^{\mu}[2,3,4,5]$. Our solutions can contain only one function depending on both time as well as overall or relative transverse space coordinates. In particular, the solutions in case (II) tell us that the brane which depends on overall transverse coordinate can be extended to the time dependent case. It is possible to get the dynamical intersecting brane solutions which obey the intersection rule $\chi=-2$ different from the usual one, as we have discussed in case (III).

We have used the intersection rules to find the cosmological solution because it is not so easy to find it analytically without their rules. The intersection rules have led to the functions $h_{r}$ and $h_{s}$ which can be written by linear combinations of terms depending on both coordinates of worldvolume and transverse space. This feature is expected to be shared by a wide class of supersymmetric solutions beyond the examples considered in the present paper, because the result has been obtained by analyzing the general structure of solutions for warped compactification with field strength of the ten- or eleven-dimensional supergravities under ansatz that is natural to include supersymmetric solutions as a special case. We have showed that these solutions give a FLRW universe if we regard the homogeneous and isotropic part of the brane worldvolumes as our spacetime. Unfortunately, the power of the scale factor is so small that the solutions of field equations cannot give a realistic expansion law. This means that we have to consider additional matter on the brane in order to get a realistic expanding universe.

The solutions we have obtained may give some moduli instabilities because of the flat direction of the moduli potential in the lower-dimensional effective theories after compactifications $[1,3,6]$. Such instability will grow unless the global or local minimum of the potential can be produced by some correction in the effective theory.

The dynamical solutions contain only one function depending on both time and transverse space coordinates. One possible reason for this is that the ansatz concerning the structure of the $D$-dimensional metric is too restrictive. However, a recent study of similar systems shows that it is possible to obtain solutions with each function depending on both time and transverse space coordinates. It is interesting to examine if our solutions can be extended to more general solutions by relaxing the assumptions of the field ansatz.

\section{References}

[1] M. Minamitsuji, N. Ohta and K. Uzawa, Phys. Rev. D 81 (2010) 126005 [arXiv:1003.5967 [hep-th]].

[2] P. Binetruy, M. Sasaki and K. Uzawa, Phys. Rev. D 80 (2009) 026001 [arXiv:0712.3615 [hep-th]].

[3] K. i. Maeda, N. Ohta and K. Uzawa, JHEP 0906 (2009) 051 [arXiv:0903.5483 [hep-th]].

[4] M. Minamitsuji, N. Ohta and K. Uzawa, Phys. Rev. D 82 (2010) 086002 [arXiv:1007.1762 [hep-th]].

[5] H. Kodama and K. Uzawa, JHEP 0507 (2005) 061 [arXiv:hep-th/0504193].

[6] H. Kodama and K. Uzawa, JHEP 0603 (2006) 053 [arXiv:hep-th/0512104]. 